Instituto de Nutrición y Tecnología de los Alimentos (INTA) Universidad de Chile, Chile.

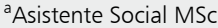

${ }^{\text {b} A d m i n i s t r a d o r a ~ P u ́ b l i c a . ~}$ 'Psicóloga MPH.

Recibido el 31 de julio de 2013, aceptado el 20 de enero de 2014.

Correspondencia a: Fernando Vio El Líbano 5524, Casilla 138, Correo 11, Santiago, Chile. Teléfono (56-2) 9781576. fvio@inta.uchile.cl

\title{
Evaluación de un programa de educación a distancia en Promoción de Salud para la Atención Primaria en Chile
}

\author{
JUDITH SALINAS ${ }^{\mathrm{a}}$, CAROLINA MUÑOZ \\ ANDREA ALBAGLI ${ }^{\circ}$, FERNANDO VIO

\section{Evaluation of an online health promotion Diploma}

\begin{abstract}
Background: Health promotion in Chile is essential, considering its current epidemiologic status where chronic diseases are predominant. Aim: To report the evaluation of an on-line Diploma in health promotion for Primary Health Care professionals. Material and Methods: Two hundred sixty four professionals from all regions of the country (40\% rural and 60\% urban) took the Diploma. It lasted 8 months with 248 academic hours distributed in 5 modules with a total of 15 units. The program was evaluated with four surveys answered by the students (general description of participants, mid-term, final and follow-up). Results: Students were highly satisfied with the program and especially with its interactive methodology which included tutorials, individual and group assignments, online discussions and a final project. The drawbacks were time restrictions and limited internet access. Ninety percent of students completed the course with an overall mean grade of 5.57 (out of a 1-7 scale). The follow-up survey showed that students implemented the new health promotion knowledge acquired, and put in practice their final assignment in their local counties. Also, most students improved their working conditions. Conclusions: The on-line interactive and participative methodology applied in this Diploma had positive results and the evaluation model can be applied in other health promotion online education programs.
\end{abstract}

(Rev Med Chile 2014; 142: 184-192)

Key words: Health education; Health information systems; Primary health care.

E

1 Instituto de Nutrición y Tecnología de los Alimentos de la Universidad de Chile (INTA) ha realizado diversos programas de educación en promoción de salud desde el año $1998^{1}$ y a distancia desde $2002^{2}$. Su finalidad es contribuir al desarrollo de capacidades y construcción de políticas públicas que enfrenten adecuadamente la realidad epidemiológica y social del país, caracterizada por una post transición avanzada con predominio de enfermedades cardiovasculares, cáncer, diabetes mellitus, obesidad y problemas de salud mental, en un contexto de inequidades ${ }^{3}$.

La renovación de la Atención Primaria de Salud (APS) impulsada por la Organización Panamericana de la Salud (OPS/OMS) ${ }^{4,5}$ y el Modelo de Atención Integral de Salud con enfoque familiar y comunitario del Ministerio de Salud (MINSAL) requieren de una acción sostenida y mancomunada para el desarrollo de competencias de los equipos de salud en prevención y promoción de salud, con el fin de dar respuestas eficaces a las 
actuales necesidades de salud de la población ${ }^{6,7}$ y contribuir al cumplimiento de los objetivos sanitarios 2010-2020 del Gobierno de Chile ${ }^{8}$. Dadas nuestras características geográficas y de centralización, es difícil y costoso para los equipos de salud actualizarse en promoción de salud, frente a lo cual, el MINSAL incorporó estos temas en el Programa de Desarrollo de Recursos Humanos en la APS Municipal (PRH/APS). Al mismo tiempo, la OPS/OMS impulsó una estrategia para el desarrollo de competencias del personal de salud, con utilización de las Tecnologías de Información y Comunicación (TIC) ${ }^{9}$.

La literatura internacional muestra el creciente uso y efectividad de la educación a distancia en el perfeccionamiento de los profesionales de la salud, señalando cambios importantes en el aprendiza$\mathrm{je}^{10,11} \mathrm{y}$ alta satisfacción de usuarios, especialmente cuando incorporan interactividad ${ }^{12,13}$ y ejercicios prácticos ${ }^{14}$. Cursos más largos muestran mejores resultados, al igual que el uso de tutorías ${ }^{15}$. El uso de programas de educación en línea está influido por la percepción de la calidad y utilidad del mismo para el desempeño laboral ${ }^{12,13,16}$ y por la adecuación de la demanda de horas de estudio a su jornada laboral ${ }^{17}$. En relación a evaluación de programas de educación a distancia en promoción de salud, no se encontraron estudios específicos en este tema. Existen estudios de evaluación en programas de educación en salud pública ${ }^{18}$ que incluyen la promoción de salud ${ }^{19}$.

El objetivo de este artículo es evaluar un Diploma de educación a distancia en promoción de salud para profesionales de la APS y aportar un modelo de evaluación para los futuros programas de formación a distancia en promoción de salud en la APS.

\section{Material y Métodos}

\section{Sujetos}

El Diploma de Postítulo a Distancia en Promoción de Salud y Calidad de Vida para equipos de APS (en adelante Diploma), se inició con 264 profesionales de 145 comunas ( $40 \%$ rurales y $60 \%$ urbanas) de las 15 regiones del país, seleccionados en los Servicios de Salud por concursos internos.

\section{Programa educativo}

El Diploma se realizó mediante convenio entre INTA, Servicios de Salud y Municipios, con financiamiento de MINSAL proveniente del PRH/ APS. La difusión se hizo por los mecanismos de comunicación de la red de salud.

El modelo educativo (Figura 1) fue de aprendizaje en red, con una metodología colaborativa,

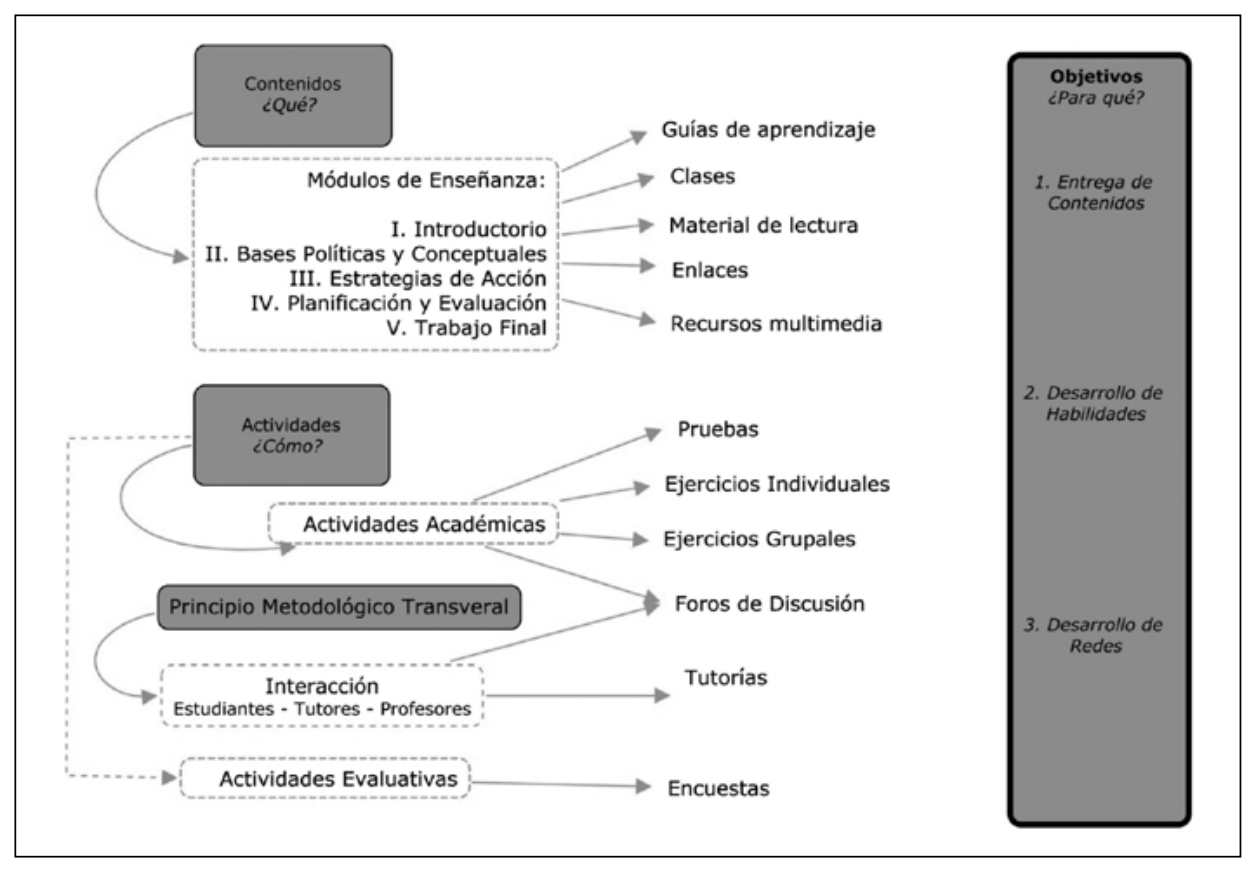

Figura 1. Modelo educativo. 
activa y participativa que privilegia el intercambio, la reflexión y el análisis grupal en la construcción del conocimiento, para la entrega de contenidos, desarrollo de habilidades y redes, lo cual puede constituir un modelo para futuros programas de educación a distancia en promoción de salud ${ }^{20-22}$.

La duración del Diploma fue de 8 meses (agosto de 2011 a abril de 2012) con 248 h académicas, y apoyo tutorial de 7 académicos con jerarquía de profesores titular, asociado y adjunto ( 4 médicos, 1 nutricionista, 1 asistente social y 1 administrador público) y 12 tutores (odontóloga, psicóloga, socióloga, ingeniero, 4 profesores, 2 nutricionistas y 2 asistentes sociales), 4 con grado de Magíster y 8 con Diplomas de postítulo, con experiencia en promoción de salud y educación a distancia.

Los contenidos se estructuraron en 5 Módulos de Aprendizaje con 15 Unidades Docentes. Las áreas temáticas fueron: Módulo I, bases pedagógicas y uso de las TIC en educación a distancia; Módulo II, bases políticas y conceptuales para una intervención efectiva en promoción de salud; Módulo III, estrategias de acción en áreas problemas y grupos de población; Módulo IV, planificación y evaluación; Módulo V, trabajo final consistente en la formulación de una intervención en promoción de salud para sus lugares de trabajo.

El Diploma usó plataforma Moodle, versión 1.9.5 estable, servidor Apache 2.2, PHP 5 del campus virtual del INTA, cuya característica es facilitar el intercambio entre los alumnos y de ellos con tutores y docentes.

\section{Evaluación del Diploma}

La recolección de datos se realizó mediante cuatro encuestas: al inicio $(\mathrm{n}=237)$, intermedia $(\mathrm{n}=179)$, al final $(\mathrm{n}=202)$ y de seguimiento $(\mathrm{n}=171)$ realizada 8 meses después de finalizado el Diploma. Las encuestas formaron parte de la metodología del Diploma dirigidas a un grupo específico, que son los estudiantes. Se midió la consistencia interna de las preguntas y las variables fueron seleccionadas de la literatura disponible sobre educación a distancia ${ }^{23-26}$. Las variables están descritas en Tabla 1.

\section{Evaluación de los estudiantes}

Las notas tuvieron una escala de 1 a 7 (mínimo de aprobación 4). Cada uno de los módulos II al V tenía una ponderación de $25 \%$ de la nota final. Las notas de los módulos II al IV se obtenían de ejercicios individuales y grupales $(40 \%)$, pruebas (30\%) y participación en foros $(30 \%)$. El módulo I era sin calificación y el módulo $\mathrm{V}$ correspondía al trabajo final.

\section{Análisis estadístico}

Se realizó un análisis descriptivo de las variables en los sujetos de la capacitación según indicadores del perfil, rendimiento y evaluación de

\section{Tabla 1. Variables del modelo de evaluación según encuestas}

\begin{tabular}{|c|c|c|}
\hline Encuesta de Inicio & Encuestas intermedia y final & Encuesta de seguimiento \\
\hline Edad, género, profesión & $\begin{array}{l}\text { Evaluación de materiales educativos y activida- } \\
\text { des académicas por módulo y unidad }\end{array}$ & $\begin{array}{l}\text { Lugar de trabajo, cargo, comuna y región } \\
\text { Cambios laborales asociados al diploma }\end{array}$ \\
\hline $\begin{array}{l}\text { Lugar de trabajo, cargo, } \\
\text { comuna y región }\end{array}$ & $\begin{array}{l}\text { Comunicación e intercambio con profesores, } \\
\text { tutores y pares }\end{array}$ & Funciones actuales en promoción de salud \\
\hline Antigüedad en la APS & $\begin{array}{l}\text { Utilidad y pertinencia de los contenidos por } \\
\text { unidad }\end{array}$ & $\begin{array}{l}\text { Adquisición y aplicación de contenidos y } \\
\text { habilidades }\end{array}$ \\
\hline $\begin{array}{l}\text { Experiencia previa en } \\
\text { educación a distancia }\end{array}$ & $\begin{array}{l}\text { Cumplimiento de objetivos de aprendizaje } \\
\text { por módulo }\end{array}$ & Implementación del trabajo final \\
\hline Expectativas & Cumplimiento de expectativas por módulo & Satisfacción y cumplimiento de expectativas \\
\hline \multirow[t]{2}{*}{ Motivación } & $\begin{array}{l}\text { Barreras en el proceso de aprendizaje } \\
\text { Valoración de la metodología y del sistema } \\
\text { de evaluación }\end{array}$ & $\begin{array}{l}\text { Barreras en la implementación de contenidos } \\
\text { Innovaciones y nuevas iniciativas }\end{array}$ \\
\hline & Acceso y navegación en la plataforma & $\begin{array}{l}\text { Comparación del diploma con otras experien- } \\
\text { cias de educación a distancia } \\
\text { Recomendación del diploma }\end{array}$ \\
\hline
\end{tabular}


los estudiantes. Para el análisis de la consistencia interna de las encuestas, se aplicó el coeficiente alfa de Cronbach ${ }^{27}$, considerándose una buena consistencia interna, cuando el valor de alfa era superior a 0,7 . Se utilizó número de casos y proporciones para resultados generales, además de media, desviación estándar, mínimo y máximo. Se calculó test de $\chi^{2}$ para comparar grupos con y sin experiencia previa en educación a distancia con STATA 10.1 para Windows.

\section{Resultados}

Encuesta descripción general de los participantes

Los resultados de la primera encuesta están descritos en la Tabla 2. El 45\% de los sujetos tenía formación de pre-grado en ciencias sociales o educación (asistentes sociales, psicólogos, educadores) y 55\% del área biomédica (nutricionistas, matronas, kinesiólogos, enfermeras, odontólogos y otros). Los alumnos tenían en promedio 35 años de edad y 8,1 años de experiencia laboral en la APS; $25 \%$ se desempeñaba en las Corporaciones de Salud Municipal y el 75\% restante en Centros de Salud Familiar (CESFAM) y Centros Comunitarios de Salud Familiar (CECOSF). El 69\% ejercía funciones de jefatura o coordinación de programas y el $31 \%$ restante eran profesionales de salud.

Para un tercio fue la primera experiencia de educación a distancia y la mayoría tenía altas expectativas sobre el Diploma. Hubo un porcentaje significativamente menor de profesionales con experiencia previa en educación a distancia en la Región Metropolitana que en las otras regiones del país $\left(\chi^{2} 14,3105\right.$ p 0,003$)$. Respecto a la responsa-

Tabla 2. Características generales de los participantes

\begin{tabular}{|c|c|c|c|}
\hline$n=264$ & & $\mathbf{n}$ & $\%$ \\
\hline \multirow[t]{2}{*}{ Género } & Mujer & 218 & 82,6 \\
\hline & Hombre & 46 & 17,4 \\
\hline \multirow[t]{5}{*}{ Edad (años) } & $20-30$ & 91 & 34,8 \\
\hline & $31-40$ & 107 & 40,5 \\
\hline & 41 y más & 66 & 25,0 \\
\hline & Promedio $\pm \mathrm{DE}$ & $35 \pm 17,5$ & \\
\hline & Rango & $23-62$ & \\
\hline \multirow[t]{9}{*}{ Profesión } & Asistente social & 76 & 28,8 \\
\hline & Nutricionista & 55 & 20,8 \\
\hline & Matrona & 32 & 12,1 \\
\hline & Psicólogo & 23 & 8,7 \\
\hline & Kinesiólogo & 19 & 7,2 \\
\hline & Enfermera & 14 & 5,3 \\
\hline & Odontólogo & 14 & 5,3 \\
\hline & Educador & 16 & 6,1 \\
\hline & Otros (médico, tecnólogo médico, terapeuta ocupacional, periodista) & 15 & 5,7 \\
\hline \multirow{4}{*}{$\begin{array}{l}\text { Región de pertenencia } \\
\text { de los participantes }\end{array}$} & Macro región norte (Arica, lquique, Antofagasta, Atacama y Coquimbo) & 32 & 12,1 \\
\hline & Macro región centro (Valparaíso, O’Higgins y Maule) & 68 & 25,8 \\
\hline & Macro región sur (Biobío, Araucanía, Los Ríos, Los Lagos y Magallanes) & 93 & 35,2 \\
\hline & Región Metropolitana & 71 & 26,9 \\
\hline \multirow{2}{*}{$\begin{array}{l}\text { Tipo de comuna de } \\
\text { trabajo }\end{array}$} & Urbana & 158 & 59,8 \\
\hline & Rural & 106 & 40,2 \\
\hline \multirow[t]{2}{*}{ Lugar de trabajo } & Municipio & 66 & 25 \\
\hline & Establecimiento de APS (CESFAM, CECOSF) & 198 & 75 \\
\hline \multirow{3}{*}{$\begin{array}{l}\text { Responsabilidad en el } \\
\text { cargo }\end{array}$} & Jefatura, encargado de programas o coordinador de sector & & \\
\hline & Profesional de equipo & 182 & 68,9 \\
\hline & & 82 & 31,1 \\
\hline \multirow{2}{*}{$\begin{array}{l}\text { Experiencia previa en } \\
\text { educación a distancia }\end{array}$} & Con experiencia & 174 & 65,9 \\
\hline & Sin experiencia & 90 & 34,1 \\
\hline
\end{tabular}


bilidad del cargo, se encontró que 77,8\% de los directivos tenían una significativa mayor experiencia previa en educación a distancia, comparado con $60,5 \%$ de los profesionales $\left(\chi^{2} 7,3732\right.$ p 0,007$)$.

\section{Encuesta intermedia}

El 86,5\% de los estudiantes encontró que los contenidos y materiales educativos eran adecuados y aportaron a su proceso de aprendizaje. El 70,8\% mencionó que los ejercicios individuales y grupales fueron de gran utilidad. El intercambio con los tutores y profesores tuvo una mejor evaluación que el realizado con los compañeros.

La actividad con mayor dificultad fue el trabajo grupal, que requiere gran coordinación entre pares, con una colaboración desigual. Los foros de discusión fueron bien evaluados, a pesar de la dificultad en su calificación.

Los obstáculos más importantes fueron la falta de tiempo y de acceso a internet. La baja conectividad se debió a la restringida conexión en algunas localidades, donde existe acceso a internet sólo en el trabajo.

\section{Resultado del diploma y encuesta final}

La tasa de aprobación de los estudiantes fue de $90,15 \%$ y las causales de reprobación fueron rendimiento $(8,7 \%)$ y postergación $(1,1 \%)$. La nota promedio final fue 5,57 (Tabla 3 ).

El 82\% de los trabajos finales estuvo relacionado con estilos de vida saludable. El 18\% restante fue en salud sexual y reproductiva, salud mental, salud bucal, participación social y medio ambiente. El 53,4\% de los profesionales realizó su trabajo final en establecimientos de educación; $18 \%$ en los propios centros de salud, y $30 \%$ restante en comunas o lugares de trabajo (Tabla 4). Los grupos destinatarios fueron pre-escolares y escolares, adolescentes y adultos mayores.

El número de horas de estudio promedio por estudiante fue de $9 \mathrm{~h}$ semanales y $74 \%$ consideró

Tabla 3. Rendimiento académico-aprobación y nota promedio

\begin{tabular}{|c|c|c|}
\hline & $\mathbf{n}$ & $\%$ \\
\hline Estudiantes matriculados & 264 & 100 \\
\hline Estudiantes aprobados & 238 & 90,2 \\
\hline Estudiantes reprobados & 23 & 8,7 \\
\hline Estudiantes postergados & 3 & 1,1 \\
\hline $\begin{array}{l}\text { Promedio } \pm \mathrm{DE} \\
\text { Rango }\end{array}$ & \multicolumn{2}{|c|}{$\begin{array}{c}5,57 \pm 1,1 \\
1-6,85\end{array}$} \\
\hline $\begin{array}{l}\text { Promedio de notas final por } \\
\text { módulos de aprendizaje }\end{array}$ & $\begin{array}{l}\text { Nota } \\
\bar{x}\end{array}$ & DE \\
\hline Modulo II Bases políticas y conceptuales & 5,56 & 1,05 \\
\hline $\begin{array}{l}\text { Modulo III Estrategias de acción e } \\
\text { intervenciones }\end{array}$ & 5,80 & 1,20 \\
\hline Modulo IV Planificación y evaluación & 5,26 & 1,38 \\
\hline Modulo V Trabajo Final & 5,60 & 1,46 \\
\hline
\end{tabular}

Tabla 4. Trabajos finales según área temática y espacio

\begin{tabular}{|c|c|c|c|c|c|c|c|c|c|c|c|c|}
\hline & \multicolumn{2}{|c|}{ Escuelas } & \multicolumn{2}{|c|}{$\begin{array}{l}\text { Centros } \\
\text { de salud }\end{array}$} & \multicolumn{2}{|c|}{ Comunas } & \multicolumn{2}{|c|}{$\begin{array}{l}\text { Lugares } \\
\text { de trabajo }\end{array}$} & \multicolumn{2}{|c|}{$\begin{array}{l}\text { Dos o más } \\
\text { espacios }\end{array}$} & \multicolumn{2}{|c|}{ Total } \\
\hline & $\mathbf{n}$ & $\%$ & $\mathbf{n}$ & $\%$ & n & $\%$ & $\mathbf{n}$ & $\%$ & $n$ & $\%$ & n & $\%$ \\
\hline Alimentación y actividad física & 81 & 63,8 & 20 & 46,5 & 16 & 45,7 & 2 & 12,5 & 11 & 64,6 & 130 & 54,6 \\
\hline Estilos de vida saludable & 6 & 4,7 & 8 & 18,6 & 7 & 20,0 & 14 & 87,5 & 2 & 11,8 & 37 & 15,5 \\
\hline Tabaco, alcohol & 20 & 15,8 & 3 & 7,0 & 3 & 8,6 & 0 & 0 & 2 & 11,8 & 28 & 11,8 \\
\hline Salud sexual y reproductiva & 13 & 10,2 & 5 & 11,6 & 0 & 0 & 0 & 0 & 2 & 11,8 & 20 & 8,4 \\
\hline Capacitación y participación & 0 & 0 & 2 & 4,7 & 6 & 17,1 & 0 & 0 & 0 & 0 & 8 & 3,4 \\
\hline Salud bucal & 5 & 3,9 & 1 & 2,3 & 0 & 0 & 0 & 0 & 0 & 0 & 6 & 2,5 \\
\hline Salud mental & 1 & 0,8 & 2 & 4,7 & 3 & 8,6 & 0 & 0 & 0 & 0 & 6 & 2,5 \\
\hline Medio ambiente & 1 & 0,8 & 2 & 4,6 & 0 & & 0 & 0 & 0 & 0 & 3 & 1,3 \\
\hline Total & 127 & 100 & 43 & 100 & 35 & 100 & 16 & 100 & 17 & 100 & 238 & 100 \\
\hline
\end{tabular}




\begin{tabular}{|c|c|c|c|c|c|}
\hline \multicolumn{6}{|l|}{ Pruebas } \\
\hline \multicolumn{6}{|l|}{ Foros de Discusión } \\
\hline \multicolumn{6}{|l|}{ Ejercicios Grupales } \\
\hline \multicolumn{6}{|l|}{ Trabajo Final } \\
\hline \multicolumn{6}{|l|}{ Ejercicios Individuales } \\
\hline & $\begin{array}{c}\text { Ejercicios } \\
\text { Individuales }\end{array}$ & Trabajo Final & $\begin{array}{l}\text { Ejercicios } \\
\text { Grupales }\end{array}$ & $\begin{array}{l}\text { Foros de } \\
\text { Discusión }\end{array}$ & Pruebas \\
\hline - \% muy de acuerdo & 76,2 & 62,4 & 44,6 & 36,1 & 26,7 \\
\hline " $\%$ de acuerdo & 23,8 & 35,2 & 45,5 & 48,5 & 55,5 \\
\hline = \% indiferente & 0 & 2,5 & 5,9 & 9,4 & 5,5 \\
\hline \% en desacuerdo & 0 & 0 & 4 & 4,5 & 11,9 \\
\hline m muy en desacuerdo & 0 & 0 & 0 & 1,5 & 0,5 \\
\hline
\end{tabular}

Figura 2. Utilidad de las actividades académicas - Encuesta de cierre $(n=202)$.

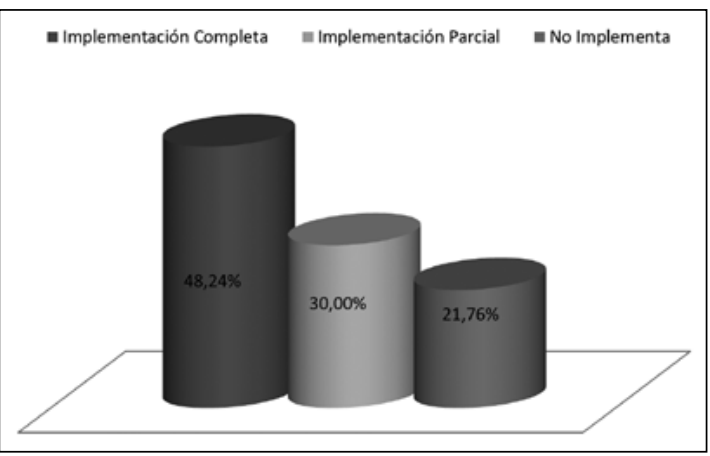

Figura 3. Implementación del trabajo final - Encuesta de seguimiento $(n=171)$.

que la plataforma virtual era fácil y amigable.

$\mathrm{Al}$ término del Diploma, los estudiantes evaluaron positivamente las unidades temáticas, el material educativo y la utilidad de las actividades académicas. Los ejercicios, foros y trabajo final tuvieron sobre $90 \%$ y las pruebas sobre $80 \%$ de evaluación positiva (Figura 2 ). En términos globales, $98 \%$ indicó que se cumplieron los objetivos y sus expectativas iniciales; $95,9 \%$ realizó un balance de aprendizaje satisfactorio y $97,7 \%$ reportó que fue pertinente y útil para su trabajo en la APS y que recomendaría este Diploma.

\section{Encuesta de seguimiento posterior}

La encuesta de seguimiento después de 8 meses de finalizado el programa tuvo una respuesta de $71,9 \%$. El $22 \%$ de los titulados señaló haber obtenido un nuevo cargo de coordinación de programa después del Diploma; 20\% tuvo aumento de sueldo o grado; $6 \%$ accedió a nuevas instancias de capacitación; $5 \%$ tuvo nota de mérito y $23 \%$ dijo haber experimentado otro tipo de cambios (mayor conocimiento, confianza y mejor atención de salud).

Antes del Diploma, 89,4\% de los alumnos realizaba actividades de promoción de salud, lo cual aumentó a $94,1 \%$ después de su término.

La aplicación de los contenidos en la práctica laboral mostró que $64,7 \%$ inició un trabajo comunitario; $60,5 \%$ introdujo mejoras en un programa de salud; $60,3 \%$ incorporó actividades de promoción en la programación local; $55,8 \%$ formuló nuevos proyectos o intervenciones en promoción y 42,9\% evaluó actividades de promoción de salud ya realizadas. Además, el 48,2\% de los encuestados implementó el trabajo final en su totalidad y $30 \%$ lo hizo parcialmente (Figura 3 ). Como causal para no implementarlo, la mitad de los encuestados señaló que en su lugar de trabajo no se priorizaba la promoción de salud.

El 82\% señaló que el Diploma les ayudó a adquirir habilidades y conocimientos nuevos y aplicar los contenidos teóricos, metodológicos y de intervenciones específicas, aumentando su confianza laboral. El 94,6\% mencionó haber cumplido con sus expectativas y de aquellos con experiencia previa en educación en línea, $62 \%$ reportó que este Diploma fue superior en calidad a otros anteriores. Por último, después de 8 meses, 97,5\% de los post graduados dijo que lo recomendaría a otras personas.

Las barreras más frecuentes para implementar los contenidos del Diploma fueron la escasez de 


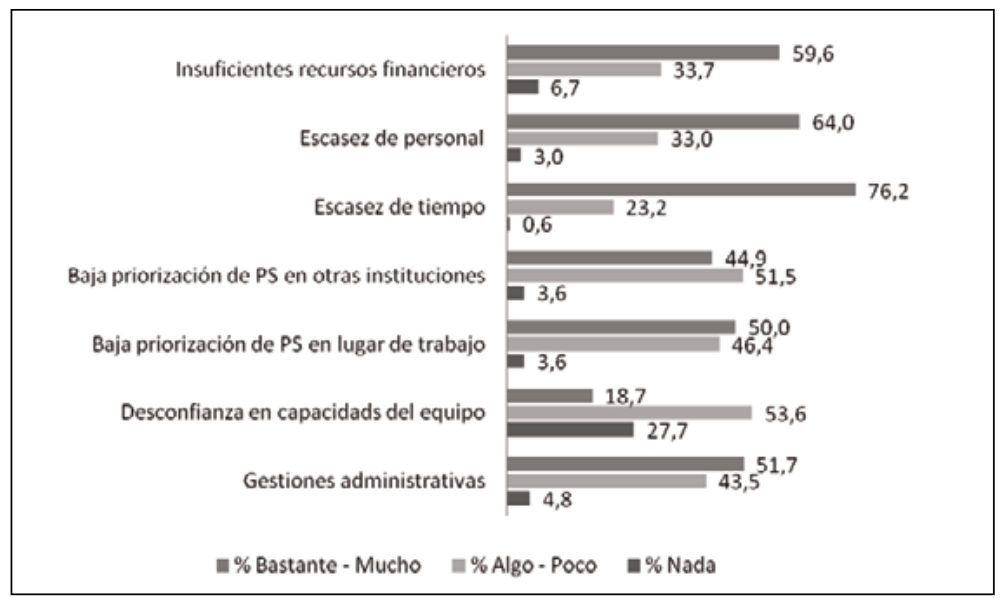

Figura 4. Barreras de implementaciónEncuesta de seguimiento $(n=171)$. Promoción de Salud (PS). tiempo $(76,1 \%)$, de personal $(64 \%)$ y la falta de recursos financieros $(59,6 \%)$ (Figura 4$)$.

\section{Discusión}

Este Diploma finalizó con una tasa de aprobación de $90,15 \%$, valor superior a $64 \%$ y $61,5 \%$ comunicado por programas de similares características ${ }^{17,28}$. Cursos de capacitación trimestral con profesionales de salud han reportado una aprobación de $86 \%{ }^{29}$ y un diploma para médicos generales $82 \%{ }^{30}$, tasas menores a la observada en esta experiencia.

El Diploma tuvo cobertura nacional, incluyendo comunas aisladas, rurales y distantes de los centros de formación, que se concentran en la Región Metropolitana. Carácter similar a experiencias de menor duración reportadas en Brasil y México ${ }^{17,31}$ con distintos niveles operativos de la red de salud y a las capacitaciones realizadas por MINSAL ${ }^{29}$.

El resultado más importante del Diploma fue la adquisición de nuevos conocimientos y habilidades en promoción de la salud y su aplicación con intervenciones específicas en el lugar de trabajo.

La experiencia previa en educación a distancia no fue predictora de un mayor rendimiento, tal vez debido a la semana de inducción en el uso de la plataforma, la metodología y la motivación de actualización. Los trabajos finales respondieron a orientaciones ministeriales y a prioridades de salud local. La mayor parte se enfocó en estilos de vida y entornos saludables, coherente con la situación epidemiológica y social del país ${ }^{3,8}$.

La evaluación de 4 encuestas en el tiempo per- mitió obtener una visión global y sistemática del proceso de enseñanza-aprendizaje y los cambios posteriores logrados en la práctica profesional. Esto permite tener un modelo de evaluación futura de educación a distancia.

Los estudiantes prefirieron los ejercicios individuales y calificaron en menor medida las pruebas, por la falta de tiempo para estudiar. La alta valoración a la interacción con tutores fue similar en las diferentes mediciones, seguido por profesores y sus pares. Esto se explica porque los tutores son el agente más activo en la comunicación personalizada y grupal con los estudiantes.

Los resultados obtenidos son consistentes con lo señalado por diferentes autores ${ }^{10,14,15,29}$, quienes demostraron que la educación continua a distancia es efectiva en transmitir conocimientos para generar cambios en el quehacer laboral. Otros factores de logro reportados en la literatura son la discusión abierta entre estudiantes, docentes y tutore $^{28}$ realizadas en los diferentes foros y trabajos grupales. Estos estudios mostraron que el uso de casos prácticos es más efectivo que el uso exclusivo de material de lectura, por la mayor interactividad y participación que generan. La falta de tiempo y acceso a internet son obstáculos descritos también por otros investigadores ${ }^{11,14,17}$.

La gran mayoría de los encuestados 8 meses después, señaló que el Diploma contribuyó a aumentar su confianza laboral, adquiriendo habilidades y conocimientos útiles y pertinentes para mejorar su práctica en la APS.

Muchos graduados accedieron a nuevos cargos con más responsabilidades e incremento de 
sueldos. Este logro refleja el reconocimiento a esta formación en los establecimientos de salud.

Las barreras para aplicar contenidos fueron de carácter organizacional, donde las limitaciones de tiempo, recursos y personal son obstáculo para el trabajo en promoción de la salud para profesionales de distinta formación, cargo y región. Se sugirió destinar horas especiales para dedicar al Diploma y un mayor compromiso de los directivos para apoyar la implementación del trabajo final.

Los resultados muestran que las limitaciones no recaen en la calidad, ni en los contenidos y procesos del Diploma, sino en la organización institucional, observándose escasa priorización de la promoción de salud y falta de voluntad política para aprovechar esta formación, tal como ha sido planteado por otros autores ${ }^{30}$.

Este estudio objetiva las ventajas de la formación a distancia, contribuyendo a promover esta forma de capacitación, que es particularmente útil en nuestro país por sus características geográficas y los altos costos que involucran las capacitaciones presenciales, tanto para los integrantes del equipo de salud, como para el sistema de salud.

\section{Referencias}

1. Salinas J, Vio F. Promoción de Salud en Chile. Rev Chil Nutr 2002; 29: 164-73.

2. Salinas J, Vio F. Educación a distancia en promoción de salud: una experiencia innovadora. Rev Med Maule 2011; 27: 10-17.

3. Vio F, Albala C, Kain J. Nutrition transition in Chile revisited: mid-term evaluation of obesity goals for the period 2000-2010. Public Health Nutr 2008; 11: 405-12.

4. OPS/OMS. La Renovación de la Atención Primaria de Salud en las Américas. Documento de Posición, 2007. Disponible en: http://cursos.campusvirtualsp.org/file, php/118/Modulo_I/md3-lp-renovacion-APS-spa-2007. pdf [Consultado el 24 de julio de 2013].

5. OPS/OMS La Renovación de la Atención Primaria de Salud en las Américas. Redes Integradas de Servicios de Salud: Conceptos, Opciones de Política y Hoja de Ruta para su Implementación en las Américas. Disponible en: http://www.paho.org/uru/index2.php?option=com docman\&task=doc_view\&gid=145\&Itemid=250 [Consultado el 24 de julio de 2013].

6. Ministerio de Salud, Chile. División de Gestión de la Red Asistencial. Modelo de Atención Integral en Salud, Serie Cuadernos Modelo de Atención $N^{\circ}$ 1, Primera Edición, diciembre 2005.

7. MINSAL, División de Atención Primaria. Orientaciones para la Planificación y Programación en Red año 2013, Septiembre 2012. Disponible en: http://www.minsal.cl portal/url/item/cd34d7da59bfbd48e0400101650154ea. pdf [Consultado el 24 de julio de 2013].

8. Gobierno de Chile, Metas 2011-2020 Elige Vivir Sano. Estrategia Nacional de Salud para el Cumplimiento de los Objetivos Sanitarios de la Década 2011-2020. Disponible en: http://www.eligevivirsano.cl/wp-content/ uploads/2012/01/Metas2011-2020.pd] [Consultado el 8 de noviembre de 2013].

9. OPS/OMS. Resolución Consejo Directivo CD50/11 Estrategia para el Desarrollo de Competencias del Personal de Salud en los Sistemas de Salud basados en la Atención Primaria de Salud, julio 2010. Disponible en http://www.campusvirtualsp.org/files/CD50-11-es.pdf [Consultado el 24 de julio de 2013].

10. Casabeer L, Kristofco R, Strasser S, Reilly M, Krishnamoorthy P, Rabin A, et al. Standardizing Evaluation of On-Line Continuing Medical Education: Physician Knowledge, Attitudes, and Reflection on Practice. JCEHP 2004; 24: 68-75.

11. Robinson T, Hills D, Kelly B. The Evaluation of an Online Orientation to Rural Mental Health Practice in Australia. J Psychiatr Ment Health Nurs 2011; 18: 62936.

12. Wong G, Greenhalgh T, Pawson R. Internet-Based Medical Education: a Realist Review of what works, for whom and in what circumstances. BMC Medical Education 2010; 46: 89-96

13. Chaney B, Eddy J, Dorman S, Glessner L, Green L, LaraAlecio R. A Primer on Quality Indicators of Distance Education. HPP 2009; 10: 222-31.

14. Cobb S. Internet Continuing Education for Health Care Professionals: An Integrative Review. JCEHP 2004; 24 : 171-80.

15. Cook D, Levinson A, Garside S, Dupras D, Erwin P, Montori V. Internet-Based Learning in the Health Professions. JAMA 2008; 10: 1181-96.

16. Ruf D, Kriston L, Berner M, Härter M. General Practitioners and Online Continuing Medical EducationWhich Factors Influence its Use? GMS 2009; 7: 1-9. Disponible en: http://www.egms.de/static/pdf/journals/ gms/2009-7/000067.pdf [Consultado el 24 de julio de 2013].

17. Sigulem D, Morais T, Cuppari L, Franceschini S, Priore S, Camargo K, et al. A Web-Based Distance Education Course in Nutrition in Public Health: Case Study. JMIR 2001; 3: e16. Disponible en: http://www.jmir.org/issue/ year/2001. [Consultado el 24 de julio de 2013]. 
18. Nartker AJ, Stevens L, Shumays A, Kalowela M, Kisimbo $\mathrm{D}$, Potter K. Increasing health worker capacity through distance learning: a comprehensive review of programmes in Tanzania. HRH 2010; 8: 30. Disponible en http:// www.lidc.org.uk/_assets/DL4H_Report_Full_reduced. pdt

19. Alexander L, Igumbor EU, Sanders D. Building capacity without disrupting health services: public health education for Africa through distance learning. HRH 2009; 7: 28 doi: 10.1186/1478-4491-7-28. Disponible en: http://www.human-resources-health. com/content $/ 7 / 1 / 28$.

20. Onrubia J. Aprender y enseñar en entornos virtuales: actividad conjunta, ayuda pedagógica y construcción del conocimiento. Disponible en: http://www.um.es ead/red/M2 [Consultado el 24 de julio de 2013].

21. Borrell RM, Lemus J, Prado O. Programa de Desarrollo del Componente Educativo en la Formación Inicial y Permanente en Salud Pública. En: Borrell RM, Rovere M. La Formación de Posgrado en Salud Pública Nuevos desafíos, Nuevos Caminos. OPS/OMS, Buenos Aires; 2004 p. 65-87.

22. Walker RM, Montero LE. Principios Generales de la Educación de Adultos. Rev Chil Med Fam 2004; 5 (2): 65-71.

23. Marcelo C. Evaluación de la calidad para programas completos de formación docente a través de estrategias de aprendizaje abierto y a distancia. RED, VII, 30 de diciembre de 2008. Disponible en: http://www.um.es/ead/ red/M7/marcelo.pdf [Consultado el 24 de julio de 2013].

24. Zapata M. Sistemas de educación a distancia a través de redes: Unos rasgos para la propuesta de evaluación de la calidad. Disponible en: http://www.um.es/ead/red/9 sistemas.pdf [Consultado el 24 de julio de 2013].

25. Hilera González J. UNE 66181: 2008, el primer estándar sobre calidad de la formación virtual. RED, VII, 30 de diciembre de 2008. Disponible en: http://www.um.es/ ead/red/M7/hilera.pdt [Consultado el 24 de julio de 2013].

26. Barberà E. Calidad de la enseñanza 2.0. RED, VII, 30 de diciembre de 2008. en: http://www.um.es/ead/red/M7 elena.pdf [Consultado el 14 de julio de 2013].

27. Cronbach LJ. Coefficient alpha and the internal structure of tests. Psychometrika 1951; 16: 297-334.

28. Zubarrew T. Educación médica a distancia: Diplomados on-line. Ars Médica 2008; 15. Disponible en http:// escuela.med.puc.cl/publ/ArsMedica/ArsMedica15/EducacionMedicaOnline.htm (consultado el 24 de julio de 2013).

29. Carabantes J, Guerra M, Guillou M. Desarrollo de un sistema de educación a distancia en el sector público de salud: 2004-2009. Rev Med Chile 2010; 138: 1148-56.

30. Montero L, Valdés V. Educación médica a distancia y experiencias en programas de educación continua a distancia. Ars Médica 2008; 15. Disponible en http:// escuela.med.puc.cl/publ/ArsMedica/ArsMedica15/ EducacionMedicaDistancia.htm (consultado el 24 de julio de 2013).

31. Magaña L, Vertiz J, Mejía MA, Rosas C, Stanford A, Molina JF, et al. Estrategia de Formación y Capacitación en Promoción de Salud: Experiencia de Educación Virtual en México. En: Arroyo H. Ed Promoción de la Salud. Modelos y experiencias de formación académicaprofesional en Iberoamérica. CIUEPS, Universidad de Puerto Rico; 2010. p. 497-518. 